\title{
Adult-onset presentation of a urea cycle disorder necessitating intensive care unit admission
}

\author{
Dustin Anderson, MD, PhD • Shailly Jain-Ghai, MD • Wendy I. Sligl, MD, \\ MSc
}

Received: 7 January 2020/Revised: 15 February 2020/Accepted: 27 February 2020/Published online: 23 March 2020

(c) Canadian Anesthesiologists' Society 2020

\section{To the Editor,}

Here we present the case of an adult male with encephalopathy, of which the underlying cause was a urea cycle disorder (UCD). His initial history revealed that he had a recent upper respiratory tract infection characterized by profound fatigue. This was followed by personality change and progressive confusion. He took no medications and had no suspicious past medical history. He maintained a vegan diet, as it apparently made him "feel better"; however, he had recently resumed eating meat, including red meat and pork.

He was a thin, ill-appearing male and was markedly diaphoretic. He was normothermic, intermittently tachycardic (80-125 beats. $\mathrm{min}^{-1}$ hypertensive (160-220/ $90-110 \mathrm{mmHg}$ ), and tachypneic ( 35 breaths $\cdot \mathrm{min}^{-1}$ ). The Glasgow Coma Scale score was 7/15. He had large reactive pupils and marked clonus to the legs but not arms. During examination, the patient had a number of 1-2-second generalized tonic-clonic seizures that spontaneously resolved.

D. Anderson, MD, PhD ( $₫)$.

Department of Critical Care Medicine, Faculty of Medicine and Dentistry, University of Alberta, Edmonton, AB, Canada

e-mail: dustin3@ualberta.ca

\section{S. Jain-Ghai, MD}

Department of Medical Genetics, Faculty of Medicine and Dentistry, University of Alberta, Edmonton, AB, Canada

W. I. Sligl, MD, MSc

Department of Critical Care Medicine, Faculty of Medicine and Dentistry, University of Alberta, Edmonton, AB, Canada

Division of Infectious Diseases, Department of Medicine, Faculty of Medicine and Dentistry, University of Alberta, Edmonton, AB, Canada
The patient received endotracheal intubation because his level of consciousness had decreased and he had ongoing seizures. Seizures were abated with a combination of intravenous lorazepam and levetiracetam. Arterial blood gas measurements showed respiratory alkalosis. Other labs of note included low urea $\left(0.9 \mathrm{mmol} \cdot \mathrm{L}^{-1}\right)$, elevated ammonia $\left(160 \mu \mathrm{mol} \cdot \mathrm{L}^{-1}\right)$, elevated international normalized ratio (2.2), and depressed fibrinogen (1.4 $\left.\mathrm{g} \cdot \mathrm{L}^{-1}\right)$. All other labs were within normal limits. Electroencephalogram (EEG) showed diffuse slowing with rare left temporal spikes (Figure A). Magnetic resonance imaging (MRI) of the brain showed left mesial frontal T2 hyperintensity (Figure B).

In the intensive care unit, mixing studies confirmed a clotting deficiency. Repeat ammonia level measurements were further elevated at $327 \mu \mathrm{mol} \cdot \mathrm{L}^{-1}$, so a UCD was considered. Intermittent hemodialysis was initiated to decrease ammonia levels. A second brain MRI (Figure C) showed worsening cerebral edema. Plasma amino acid measurements showed decreased levels of arginine and citrulline, while urine organic acid measurements revealed increased levels of orotic acid. Further therapy included the ammonia scavenging medications glycerol phenylbutyrate and I-citrulline, in addition to a high-fat, protein-limited diet. Hyperammonemia was rapidly resolved with these therapies and the patient was liberated from mechanical ventilation on post-admission day 4. Repeat EEG and MRI of the brain were normal (not shown). The patient was discharged to the neurology service on post-admission day 11. After a period of convalescence, the patient was discharged home at baseline function (post-admission day 26). At a later date, genetic testing confirmed a hemizygous variant in the ornithine transcarbamylase (OTC) gene, consistent with X-linked OTC deficiency. 


\section{Figure A)}

Electroencephalogram showing severe, asymmetric slowing, in keeping with a diffuse encephalopathy. Magnetic resonance imaging on postadmission day 0 ; B) and postadmission day 3; C) showing increasing $\mathrm{T} 2$ hyperintensities in mesial frontal structures and insular cortices, along with increasing cerebral edema.

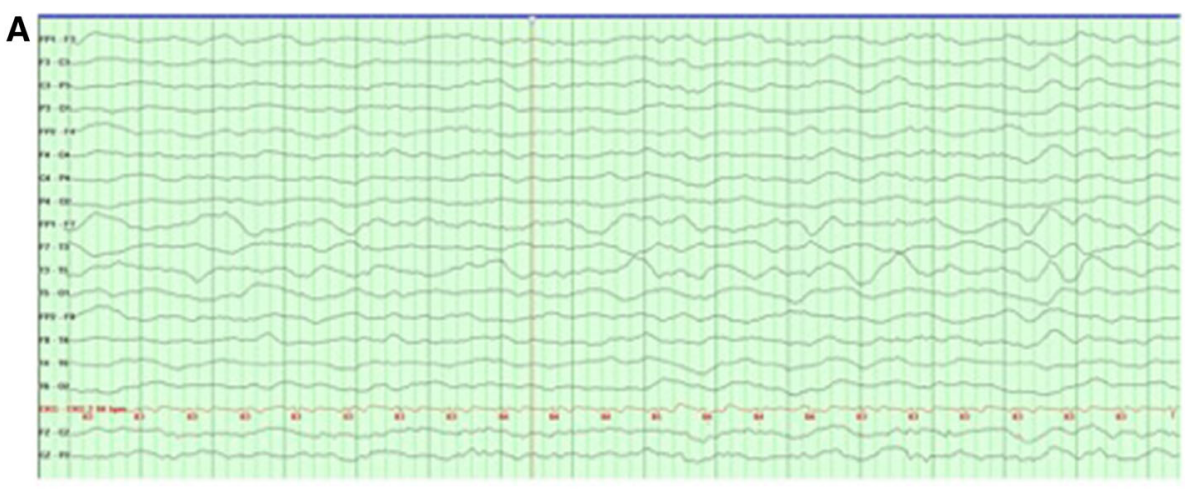

B

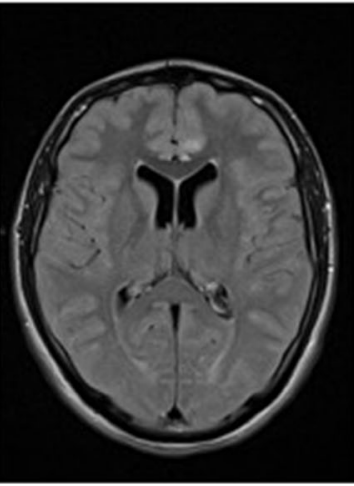

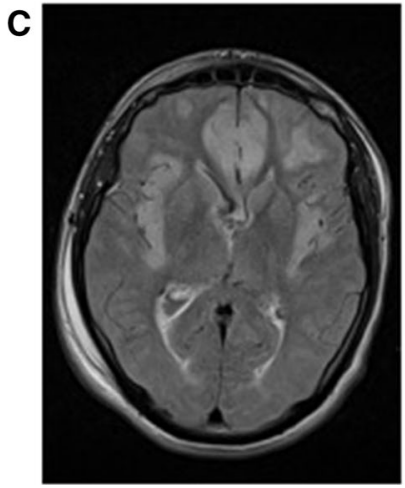

Urea cycle disorders result from defects in the urea cycle enzymes - a pathway that metabolizes nitrogenous waste. ${ }^{1}$ The incidence of UCDs is approximately 1 in 35,000 births. ${ }^{1}$ OTC deficiency is the most common UCD, accounting for approximately $55 \%$ of all UCDs. ${ }^{1}$ The majority of UCDs present either in the neonatal or childhood period, with vomiting, anorexia, and progressive encephalopathy. ${ }^{1}$ Less than $10 \%$ of UCDs present in adulthood, with symptoms including nausea, vomiting, seizures, encephalopathy, and coma. ${ }^{2}$ One important clinical clue to the diagnosis of a UCD is the presence of hyperventilation. ${ }^{1}$ Risk factors for UCD precipitation include nutritional changes (as in this case), medications that alter protein catabolism, and stress (also in this case). ${ }^{2}$

Elevated ammonia concentration (often $>100$ $\left.\mu \mathrm{mol} \cdot \mathrm{L}^{-1}\right)$ is the hallmark of UCDs. ${ }^{2}$ Urea concentrations are often low, typically from inhibition of ureagenesis. ${ }^{1}$ Arterial blood gases often show respiratory alkalosis. ${ }^{1}$ Rarely, and specific to OTC deficiency, coagulopathy develops. ${ }^{3}$ Plasma amino acids and urine organic acids help identify the specific UCD; however, definitive diagnosis of a particular enzyme deficiency necessitates genetic testing. ${ }^{1,2}$

Urea cycle disorder patients in crisis require urgent treatment with hemodialysis. Rapid initiation is important, as the neurologic outcome of UCDs depends on the duration of exposure and the degree of hyperammonemia. ${ }^{4}$ Nitrogen-scavenging agents also help ameliorate hyperammonemia and are associated with increased survival. $^{5}$ Avoidance of a catabolic state is critical, particularly acutely. Avoidance can be achieved with nutritional supplementation under the guidance of dieticians. If a UCD is especially recalcitrant, then liver transplantation may be required. ${ }^{1,2,4}$ Key principles of UCD management are summarized in guidelines from Häberle et $a l^{4}$

Acknowledgement Dr. Peter Brindley for reviewing the final manuscript.

Conflict of interest None.

Funding statement None.

Editorial responsibility This submission was handled by Dr. Sangeeta Mehta, Associate Editor, Canadian Journal of Anesthesia.

\section{References}

1. Blair NF, Cremer PD, Tchan MC. Urea cycle disorders: a lifethreatening yet treatable cause of metabolic encephalopathy in adults. Pract Neurol 2015; 15: 45-8.

2. Stepien KM, Geberhiwot T, Hendriksz CJ, Treacy EP. Challenges in diagnosing and managing adult patients with urea cycle disorders. J Inherit Metab Dis 2019; 42: 1136-46. 
3. Ihara K, Yoshino M, Hoshina T, et al. Coagulopathy in patients with late-onset ornithine transcarbamylase deficiency in remission state: a previously unrecognized complication. Pediatrics 2013; 131: e327-30.

4. Häberle J, Boddaert N, Burlina A, et al. Suggested guidelines for the diagnosis and management of urea cycle disorders. Orphanet $\mathbf{J}$ Rare Dis 2012. DOI: https://doi.org/10.1186/1750-1172-7-32.
5. Enns GM, Berry SA, Berry GT, Rhead WJ, Brusilow SW, Hamosh $A$. Survival after treatment with phenylacetate and benzoate for urea-cycle disorders. N Engl J Med 2007; 356: 2282-92.

Publisher's Note Springer Nature remains neutral with regard to jurisdictional claims in published maps and institutional affiliations. 